PROCEEDINGS OF THE

AMERICAN MATHEMATICAL SOCIETY

Volume 131, Number 11, Pages 3601-3606

S 0002-9939(03)06882-5

Article electronically published on April 1, 2003

\title{
CONDENSATIONS OF PROJECTIVE SETS ONTO COMPACTA
}

\author{
HENRYK MICHALEWSKI \\ (Communicated by Alan Dow)
}

\begin{abstract}
For a coanalytic-complete or $\Pi_{2}^{1}$-complete subspace $X$ of a Polish space we prove that there exists a continuous bijection of $X$ onto the Hilbert cube $[0,1]^{\mathbb{N}}$. This extends results of Pytkeev. As an application of our main theorem we give an answer to some questions of Arkhangelskii and Christensen.

Under the assumption of Projective Determinacy we also give some generalizations of these results to higher projective classes.
\end{abstract}

\section{INTRODUCTION}

If $X, Y$ are topological spaces then a continuous bijection $f: X \rightarrow Y$ is called a condensation. For a topological space $X$, existence of a compact space $K$ and a condensation $f: X \rightarrow K$ is equivalent to existence of a weaker compact topology on $X$.

Pytkeev proved ([8]) that if $X$ is a Borel subspace of a Polish space and $X$ is not $\sigma$-compact, then there exists a condensation of $X$ onto $[0,1]^{\mathbb{N}}$. Banach in the Scottish Book (9]) asked if there is a condensation of every separable Banach space onto a compact, metrizable space; Kulpa noticed ([5]) that an affirmative answer to this question follows from Pytkeev's theorem. One of the aims of this paper (Theorem 3) is to prove an analog of the theorem of Pytkeev for the classes of coanalytic and $\prod_{2}^{1}$-spaces; under the additional assumption of Projective Determinacy we also prove a generalization of Pytkeev's result to higher projective classes.

Christensen proved ([3]) that for a given metrizable, $\sigma$-compact space $X$ the space $C_{p}(X)=\left\{f \in \mathbb{R}^{X}: f\right.$ continuous $\}$ of all continuous functions on $X$ with topology inherited from $\mathbb{R}^{X}$ is Borel-isomorphic with the Hilbert cube $[0,1]^{\mathbb{N}}$. Moreover, Christensen noticed that for the space $\mathcal{N}$ of all irrationals, the space $C_{p}(\mathcal{N})$ is not Borel-isomorphic with $[0,1]^{\mathbb{N}}$ and asked if there is a weaker Borel structure on $C_{p}(\mathcal{N})$ which would be Borel isomorphic with $[0,1]^{\mathbb{N}}$.

Arkhangelskii proved ([2]) that if $X$ is a $\sigma$-compact metrizable space, then $C_{p}(X)$ has a weaker compact metrizable topology. He formulated a conjecture ([2], p. 1882 ) that there is no such weaker topology for the space $C_{p}(\mathcal{N})$. We prove (see the Corollary) that $C_{p}(\mathcal{N})$ has a weaker compact metrizable topology; to be more precise, we prove that $C_{p}(\mathcal{N})$ can be bijectively mapped onto the Hilbert cube

Received by the editors December 12, 2001 and, in revised form, May 21, 2002.

2000 Mathematics Subject Classification. Primary 54C35, 03E15, 28A05, 54H05.

Key words and phrases. Function spaces, topology of pointwise convergence, condensation, coanalytic set, projective set, uniformization.

The author's research was partially supported by KBN Grant 5PO3A02321. 
$[0,1]^{\mathbb{N}}$. The paper is divided into four parts. After the Introduction there is a section where we fix notation, define notions and formulate auxiliary facts from descriptive set theory. Next we prove The Main Theorem (Theorem 3) which says that every set which is coanalytic-complete or $\prod_{2}^{1}$-complete can be mapped onto $[0,1]^{\mathbb{N}}$. Under the assumption of Projective Determinacy we extend the statement of the theorem to the case of $\prod_{n}^{1}$-complete sets, $n \geq 3$. Theorem 3 is preceded by a Lemma on extensions of injective, continuous maps.

In the fourth section, we give a Corollary, which is based on results of Andretta and Marcone ([1) and our main theorem. The Corollary contains a solution of the question of Christensen and hypothesis of Arkhangelskii. More precisely, examples of coanalytic-complete sets are shown in [1] and among them there is $C_{D}(\mathcal{N})$, the projection of the space $C_{p}(\mathcal{N})$ onto $D$ coordinates, where $D$ is a fixed countable dense subset of $\mathcal{N}$. The image of the projection is a subspace of a separable and metric space $\mathbb{R}^{D}$. The projection is a bijective, continuous map. Due to our Theorem 3, we infer that there exists a bijective, continuous map from the space $C_{D}(\mathcal{N})$ onto $[0,1]^{\mathbb{N}}$. The composition of the projection and the condensation of $C_{D}(\mathcal{N})$ onto $[0,1]^{\mathbb{N}}$ gives a condensation of $C_{p}(\mathcal{N})$ onto $[0,1]^{\mathbb{N}}$. In fact we prove a more general statement: instead of $\mathcal{N}$ one may take an analytic set; under the additional assumption of Projective Determinacy, one may take a projective set.

\section{Notation AND SOME AUXILIARY FACTS}

Non-metrizable spaces appear only in the Introduction and in the Corollary. In other sections all spaces under consideration are metrizable and separable. Throughout the paper descriptive set theory is applied only to subspaces of Polish spaces.

The symbol $I$ stands for the unit interval $[0,1]$. We say that $X$ is in the class $\Sigma_{1}^{1}$ or, equivalently, that $X$ is analytic, if there exists a continuous function from irrationals $\mathcal{N}$ onto $X$.

If $X$ is a subspace of a Polish space $Y$, then we say that $X$ is in the class $\Pi_{1}^{1}$ or, equivalently, that $X$ is coanalytic, if the complement of $X$ in $Y$ is analytic. Continuous images of $\boldsymbol{\sim}_{1}^{1}$ sets are called $\boldsymbol{\Sigma}_{2}^{1}$ sets, their complements are called $\boldsymbol{\sim}_{2}^{1}$; for every $n \in \mathbb{N}$ we inductively define in the same way $\underset{\sim}{\Sigma_{n}^{1}}$ and $\underset{\sim}{\prod_{n}^{1}}$. All these classes are called the projective classes.

Whenever we use the symbol $\Gamma$, we assume that is one of the class $\underset{\sim}{\prod_{n}^{1}}$ or $\underset{\sim}{\boldsymbol{\Sigma}_{n}^{1}}$ $(n \geq 1)$. For a space $X$ the symbol $\Gamma(X)$ stands for $\{A \subset X: A \in \Gamma\}$. The projective classes $\boldsymbol{\Sigma}_{n}^{1}, \Pi_{n}^{1}$, where $n>0, n \in \mathbb{N}$, are closed with respect to the closed subsets, countable unions and intersections, homeomorphic images and continuous preimages.

We say that a subset $X$ of a Polish space $Y$ is $\Gamma$-hard (see 4], Definition 22.9) if for every set $C \in \Gamma\left(2^{\mathbb{N}}\right)$ there exists a continuous map $f: 2^{\mathbb{N}} \rightarrow Y$ such that $C=f^{-1}[X]$. We will need more information on the function $f$; namely that there exists an injection $f: 2^{\mathbb{N}} \rightarrow Y$ such that $x \in C$ if and only if $f(x) \in X$. Construction of such an injection from a given continuous mapping is the subject of a theorem of Louveau and Saint-Raymond ([7], also Exercise 26.11 of [4]). Let us notice that the theorem implies

Remark (Louveau, Saint-Raymond). If $X$ is $\Gamma$-hard, then $X$ contains closed copies of all $\Gamma$ subsets of the Cantor set. 
We say that $X$ is $\Gamma$-complete if $X$ is $\Gamma$-hard and $X \in \Gamma$. We will need the following

Theorem 1 (Moschovakis, Corollary 39.9 in [4). Let us assume Projective Determinacy and let $A$ belong to the class $\underset{\sim}{\Sigma_{n}^{1}}, n \geq 3, n \in \mathbb{N}$. Then there exist a $\prod_{n}^{1}$ subset $C$ of the Cantor set and a continuous bijection from $C$ onto $A$.

Proof. The original result of Moschovakis says (Corollary 39.9, [4]) that under the assumption of Projective Determinacy the classes $\boldsymbol{\Sigma}_{2 k+2}^{1}$ and $\prod_{2 k+1}^{1}(k \in \mathbb{N})$ have the uniformization property. Let $A$ be a subset of a Polish space $Y, A \in \boldsymbol{\Sigma}_{n}^{1}(Y)$ $(n \geq 3, n \in \mathbb{N})$. There exist $C_{0} \in \mathbb{\sim}_{n-1}^{1}\left(2^{\mathbb{N}}\right)$ and a continuous function $f: C_{0} \rightarrow Y$ such that $A=f\left[C_{0}\right]$. Let us define a homeomorphism $F: C_{0} \rightarrow Y \times 2^{\mathbb{N}}$ by the formula $F(x)=(f(x), x)$. The set $G_{0}=\left\{(y, x) \in Y \times 2^{\mathbb{N}}: f(x)=y\right\}=F\left[C_{0}\right]$ is homeomorphic to $C_{0}$, hence belongs to $\prod_{n-1}^{1}\left(Y \times 2^{\mathbb{N}}\right)$. According to Moschovakis' theorem, one of the classes $\prod_{n-1}^{1},{\underset{\sim}{n}}_{n}^{1}$ has the uniformization property; in particular, there exists $G_{1} \in \boldsymbol{\Pi}_{n}^{1}\left(Y \times 2^{\mathbb{N}}\right), G_{1} \subset G_{0}$, such that for all $y \in A$ there exists a unique $x \in 2^{\mathbb{N}}$, such that $(y, x) \in G_{1}$. We define $C_{1}=F^{-1}\left[G_{1}\right] \subset 2^{\mathbb{N}}$. Since it is a homeomorphic image of $G_{1}$, the set $C_{1}$ belongs to ${\underset{\sim}{n}}_{n}^{1}$. As we selected exactly one point from every fiber of $f$, the function $f$ restricted to $C_{1}$ is injective and $A=f\left[C_{1}\right]$.

In the case of $\underset{\sim}{\Sigma_{1}^{1}}$ and $\underset{\sim}{\boldsymbol{\Sigma}_{2}^{1}}$ sets the assumption of Projective Determinacy is not necessary:

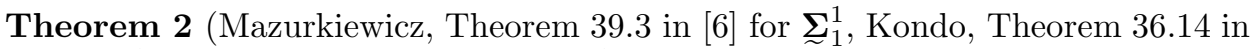
[4] for $\left.\boldsymbol{\Sigma}_{2}^{1}\right)$. Let $A$ belong to the class ${\underset{\sim}{\Sigma}}_{2}^{1}$. Then there exist a coanalytic subset $C$ of the Cantor set and a continuous bijection from $C$ onto $A$.

\section{THE MAIN THEOREM}

In this section our reasoning follows Pytkeev's ideas from [8]. Let $m$ be a fixed natural number. We define

$$
C_{m}=\left\{x \in I^{\mathbb{N}}: \text { there exists } n_{0} \text { such that } x_{n}=\frac{1}{m+1} \text { for all } n \geq n_{0}\right\} .
$$

Let $\pi_{k}$ denote the projection on the $k-$ th $(k \in \mathbb{N})$ axis of the product $I^{\mathbb{N}}$. Let $\pi_{k}^{1}, \pi_{k}^{2}(k \in \mathbb{N})$ denote projections on the appropriate axis of the product $I^{\mathbb{N}} \times I^{\mathbb{N}}$.

Lemma. Let $\Gamma$ be one of the classes $\underset{\sim}{\Sigma_{n}^{1}}, \prod_{n}^{1}(n \geq 1)$, let $X$ be in $\Gamma$, let $M \subset X$ be a closed subspace, and let $f$ be a continuous injective function $f: M \rightarrow I^{\mathbb{N}} \times\left(I^{\mathbb{N}} \backslash C_{m}\right)$ such that the image $f[M]$ is in $\Gamma\left(I^{\mathbb{N}} \times I^{\mathbb{N}}\right)$. Then there exists $g: X \rightarrow I^{\mathbb{N}} \times I^{\mathbb{N}}$, a continuous injective function which extends $f$ and such that $g(X \backslash M) \subset I^{\mathbb{N}} \times C_{m}$. Moreover, the image $g[X]$ is in the family $\Gamma\left(I^{\mathbb{N}} \times I^{\mathbb{N}}\right)$.

Proof (we use a method from [5]). Let $h$ be a homeomorphic embedding of $X$ into $I^{\mathbb{N}}$ such that for every $k \in \mathbb{N}$ the set $\left\{n \in \mathbb{N}: \pi_{n} \circ h=\pi_{k} \circ h\right\}$ is infinite. Let $M_{k}(k \in \mathbb{N})$ be a family of closed subsets of $X$ such that $M_{k} \subset M_{k+1}$ and $\bigcup_{k \in \mathbb{N}} M_{k}=X \backslash M$. For a number $k \in \mathbb{N}$ we define

$$
\begin{gathered}
h_{k}^{1}: M_{k} \cup M \rightarrow[0,1], \\
h_{k}^{2}: M_{k} \cup M \rightarrow[0,1]
\end{gathered}
$$


by the following formulas:

$$
\begin{aligned}
& h_{k}^{1}(x)= \begin{cases}\pi_{k}^{1}(f(x)) & \text { for } x \in M, \\
\pi_{k}(h(x)) & \text { for } x \in M_{k},\end{cases} \\
& h_{k}^{2}(x)= \begin{cases}\pi_{k}^{2}(f(x)) & \text { for } x \in M, \\
\frac{1}{m+1} & \text { for } x \in M_{k} .\end{cases}
\end{aligned}
$$

Let $g_{k}^{1}, g_{k}^{2}$ be any continuous functions on $X$ which extend the functions $h_{k}^{1}, h_{k}^{2}$ $(k \in \mathbb{N})$. We define $g^{1}$ and $g^{2}$ as diagonal products of $g_{k}^{1}$ and $g_{k}^{2}(k \in \mathbb{N})$ and the function $g$ as the diagonal product of $g_{1}$ and $g_{2}$. It is reassured by the definition that the function $g$ is an extension of $f$.

Let $k$ be a natural number. For every $l \in \mathbb{N}$ we fix a number $n_{l}>k$ such that $\pi_{n_{l}} \circ h=\pi_{l} \circ h$. The diagonal product of $\left\{g_{n_{l}}^{1}\right\}_{l \in \mathbb{N}}$ restricted to $M_{k}$ is equal to a homeomorphic embedding of $M_{k}$ into $I^{\mathbb{N}}$. This implies that $g$ (which is the diagonal product of a family of functions which contains all $\left\{g_{n_{l}}^{1}\right\}_{l \in \mathbb{N}}$ ) restricted to $M_{k}$ is also a homeomorphic embedding. Since the class $\Gamma$ is closed with respect to homeomorphisms and countable unions and the image $f[M]$ is in $\Gamma$, we obtain that the image $g[X]=g\left[\bigcup_{k \in \mathbb{N}} M_{k} \cup M\right]=\bigcup_{k \in \mathbb{N}} g\left[M_{k}\right] \cup f[M]$ is also in $\Gamma$.

Let us check that the image under $g$ of $X \backslash M$ is a subset of $I^{\mathbb{N}} \times C_{m}$. If $x \in X \backslash M$, then $x \in M_{k_{0}}$ for some $k_{0} \in \mathbb{N}$. For every $k \geq k_{0}(k \in \mathbb{N})$ we have $h_{k}^{2}(x)=\frac{1}{m+1}$. This implies that $g(x) \in I^{\mathbb{N}} \times C_{m}$.

In the end we should check the injectivity of $g$. Let us notice that it follows from:

- the fact that the family $\left\{M_{k}\right\}_{k \in \mathbb{N}}$ is increasing,

- the fact that $g$ restricted to $M_{k}(k \in \mathbb{N})$ and $g$ restricted to $M$ is injective and

- the fact that $g(M) \cap g(X \backslash M)=\emptyset$.

For a given space $X$ we say that a family of subsets $X_{i} \subset X(i \in I)$ is discrete if for every $x \in X$ there exists an open set $U \subset X, x \in U$ such that $U$ intersects at most one set from the family $\left\{X_{i}\right\}_{i \in I}$. We say that a family of subsets $X_{i} \subset X(i \in$ $I)$ is locally finite if for every $x \in X$ there exists an open set $U \subset X, x \in U$ such that $\left\{i \in I: X_{i} \cap U \neq \emptyset\right\}$ is a finite set.

Theorem 3. If $X$ is $\prod_{1}^{1}$-complete or $\prod_{2}^{1}$-complete, then there exists a continuous injective map from $X$ onto $I^{\mathbb{N}} \times I^{\mathbb{N}}$. Under Projective Determinacy the same holds for $X$ which is $\prod_{n}^{1}$-complete $(n \geq 3)$.

Proof of Theorem 3. Let $n>0, n \in \mathbb{N}$, be a fixed natural number and let $Y$ be any $\mathbb{I}_{n}^{1}$-complete subset of the Cantor set (one can find examples of such coanalytic sets in Chapter 27 of [4]; universal sets are standard examples of $\prod_{n}^{1}$-complete sets, $n>0, n \in \mathbb{N}$ ). Due to the fact that $X$ is $\mathbb{\sim}_{n}^{1}$-complete and the fact that the family of all $\prod_{n}^{1}$ subsets of the Cantor set is closed with respect to the multiplication by $\mathbb{N}$, there exists a closed embedding of $i: \mathbb{N} \times Y \rightarrow X$. We define $D_{k}=i[\{k\} \times Y]$ $(k \in \mathbb{N})$.

The family $\left\{D_{k}\right\}_{k \in \mathbb{N}}$ is a discrete family of $\prod_{n}^{1}$-complete sets. In particular, according to the Remark (see Section 2), for every $k \in \mathbb{N}$ the set $D_{k}$ contains closed copies of all subsets of the Cantor set which belong to the class $\mathbb{\sim}_{n}^{1}$.

Let $\left\{F_{k}: k \in \mathbb{N}\right\}$ be a locally finite covering of $X$ consisting of closed subsets of $X$ such that $D_{k} \subset F_{k} \backslash \bigcup_{l<k} F_{l}(k \in \mathbb{N})$. We define $A_{k}$ as $\bigcup_{l \leq k} F_{l}(k \in \mathbb{N})$; let 
$B_{k}=I^{\mathbb{N}} \times\left(I^{\mathbb{N}} \backslash \bigcup_{l>k} C_{l}\right)(k \in \mathbb{N})$. Let us notice that $\bigcup_{k \in \mathbb{N}} B_{k}=I^{\mathbb{N}} \times I^{\mathbb{N}}$ and $B_{k+1}=B_{k} \cup I^{\mathbb{N}} \times C_{k+1}(k \in \mathbb{N})$. We will construct a sequence of functions $f_{k}$ $(k \in \mathbb{N})$ such that

(1) $f_{k}: A_{k} \rightarrow I^{\mathbb{N}} \times I^{\mathbb{N}}$,

(2) the image $f_{k}\left[A_{k}\right]$ is in $\prod_{n}^{1}\left(I^{\mathbb{N}} \times I^{\mathbb{N}}\right)$,

(3) $f_{k+1}$ extends $f_{k}$,

(4) $B_{k} \subset f_{k+1}\left[A_{k+1}\right] \subset B_{k+1}$ and $f_{0}\left[A_{0}\right] \subset B_{0}$.

The union of the functions $f_{k}$ will be the required bijection of $X$ onto $I^{\mathbb{N}} \times I^{\mathbb{N}}$. We will start the induction from the definition of the function $f_{0}$. Let $h$ be a homeomorphic embedding of $X$ into $I^{\mathbb{N}}$ and let $f_{0}: A_{0} \rightarrow I^{\mathbb{N}} \times I^{\mathbb{N}}$ be the diagonal product of the restriction of $h$ to $A_{0}$ and the constant function equal to $(1,1, \ldots) \in$ $I^{\mathbb{N}}$. Since the homeomorphic image $f_{0}\left[A_{0}\right]$ is in $\prod_{n}^{1}\left(I^{\mathbb{N}} \times I^{\mathbb{N}}\right)$ and $f_{0}\left[A_{0}\right] \subset B_{0}$, the function $f_{0}$ fulfills all the inductive requirements.

Let $k$ be a natural number and let us assume that we constructed $f_{k}$ with the properties (1)-(4). Since $f_{k}\left[A_{k}\right]$ is in $\prod_{n}^{1}\left(I^{\mathbb{N}} \times I^{\mathbb{N}}\right)$ and $B_{k}$ is a Borel set, the difference $B_{k} \backslash f_{k}\left[A_{k}\right]$ is in $\Sigma_{n}^{1}\left(I^{\mathbb{N}} \times I^{\mathbb{N}}\right)$.

According to the Mazurkiewicz-Kondo theorem (for $n=1,2$ ) or the Moschovakis theorem (for $n \geq 3$ ), there exist a subspace $C$ of the Cantor set which is in the class $\prod_{n}^{1}\left(2^{\mathbb{N}}\right)$ and an injective continuous mapping $g$ from $C$ onto $B_{k} \backslash f_{k}\left[A_{k}\right]$. Since $F_{k+1}$ contains as a closed subset a $\prod_{n}^{1}$-complete set $D_{k+1}$, due to the Remark (see Section 2) we may assume that $C$ is a closed subset of $F_{k+1}$.

Let us define $f_{k}^{\prime}: C \cup A_{k} \rightarrow I^{\mathbb{N}} \times I^{\mathbb{N}}$ as the common extension of $f_{k}$ and $g$. Since the image $f_{k}^{\prime}\left[C \cup A_{k}\right]$ is equal to $B_{k}$, it follows that $f_{k}^{\prime}\left[C \cup A_{k}\right]$ is Borel and $f_{k}^{\prime}\left[C \cup A_{k}\right]$ is a subset of $I^{\mathbb{N}} \times\left(I^{\mathbb{N}} \backslash C_{k+1}\right)$. We apply the Lemma for $m=k+1$ to the function $f_{k}^{\prime}$ and define $f_{k+1}: A_{k+1} \rightarrow I^{\mathbb{N}} \times I^{\mathbb{N}}$ as the extension of $f_{k}^{\prime}$ given by the Lemma.

Only condition (4) may need some explanation. Since the image of the function $f_{k}^{\prime}$ is equal to $B_{k}$, the image of $f_{k+1}$ contains $B_{k}$ as well. This proves that the first inclusion holds. Due to the Lemma the image of the extension is contained in the union of the set $I^{\mathbb{N}} \times C_{k+1}$ and the image of $f_{k}^{\prime}\left[C \cup A_{k}\right]=B_{k}$. According to the definition of the family $\left\{B_{k}\right\}_{k \in \mathbb{N}}$ the union is equal to $B_{k+1}$. This proves that the second inclusion holds.

\section{Corollary}

Let $A$ be a projective subspace of a Polish space and let us assume that $A$ is not $\sigma$-compact. For analytic $A$ we set $n=1$ and otherwise $n \in \mathbb{N}$ is the natural number such that $A \in \boldsymbol{\Sigma}_{n}^{1}$ but $A \notin \boldsymbol{\Sigma}_{n-1}^{1}$.

Let us fix a metric $d$ on $A$. The symbol $C_{p}(A)$ stands for the space of all continuous real-valued functions on $A$ with the topology of pointwise convergence. We prove that $C_{p}(A)$ may be condensed onto $I^{\mathbb{N}}$; we assume Projective Determinacy unless $A$ is analytic. Let $D$ be a fixed dense countable subset of $A$. The symbol $\pi_{D}$ stands for the projection of $\mathbb{R}^{\mathcal{N}}$ onto $\mathbb{R}^{D}$. We define

$$
\begin{aligned}
C_{D}(A) & =\left\{f \in \mathbb{R}^{D}: f \text { is a restriction of a function from } C_{p}(A)\right\} \\
& =\pi_{D}\left[C_{p}(A)\right] .
\end{aligned}
$$

It was proved by Andretta and Marcone (Lemma 2.3 of [1]) that if $A$ is ${\underset{\sim}{\boldsymbol{N}}}_{n}^{1}$, then $C_{D}(A)$ is a $\prod_{n}^{1}$ subset of $\mathbb{R}^{D}$. Let us notice that $\pi_{D} \mid C_{p}(A)$ is a condensation of the space $C_{p}(A)$ onto the space $C_{D}(A)$. 
It is enough to check that $C_{D}(A)$ fulfills the assumptions of Theorem 3. It follows from results of Andretta and Marcone, who proved that the set $C_{D}(A)$ is a $\underset{\sim}{\prod_{n}^{-}}$ complete subset of the Polish space $\mathbb{R}^{D}$ ([1], Theorem 3.3 for analytic $A$, Theorem 4.3 for projective $A$ ).

\section{ACKNOWLEDGEMENTS}

I would like to express my deep gratitude to my supervisor Witold Marciszewski for many valuable discussions on the subject.

\section{REFERENCES}

1. A. Andretta, A. Marcone, Pointwise convergence and the Wadge hierarchy, Comment. Math. Univ. Carolinae 42 (2001), 159-172. MR 2002c:03073

2. A.V. Arkhangelskii, On condensations of $C_{p}$-spaces onto compacta, Proc. Amer. Math. Soc. 128 (2000), 1881-1883. MR 2001c:54002

3. J.P.R. Christensen, Topology and Borel Structure, North-Holand, Amsterdam, 1974. MR 50:1221

4. A.S. Kechris, Classical Descriptive Set Theory, Springer-Verlag, New York, 1995. MR 96e:03057

5. W. Kulpa, On a problem of Banach, Colloquium Math. 56 (1988), 255-261. MR 91d:54023

6. K. Kuratowski, Topology, vol. I, New York, 1966. MR 36:840

7. A. Louveau, J. Saint-Raymond, Borel classes and closed games: Wadge-type results and Hurewicz-type results, Trans. Amer. Math. Soc. 304 (1987), 431-467. MR 89g:03068

8. E.G. Pytkeev, The upper bounds of topologies (in Russian), Mat. Zametki 20 no. 4 (1976), 489-500. MR 55:1262

9. The Scottish Book, ed. R.D. Mauldin, Birkhauser, Boston, 1981. MR 84m:00015

Institute of Mathematics, Warsaw University, S. Banach 2 St., 02-097 Warsaw, POLAND

E-mail address: henrykm@mimuw.edu.pl 